\title{
A step towards non-invasive characterization of the human frontal eye fields of individual subjects
}

\author{
Andreas A loannides ${ }^{1 *}$, Peter BC Fenwick ${ }^{1,2}$, Elina Pitri ${ }^{1}$, Lichan Liu ${ }^{1}$ \\ From Consciousness and its Measures: Joint Workshop for COST Actions NeuroMath and Consciousness \\ Limassol, Cyprus. 29 November - 1 December 2009
}

\begin{abstract}
Background: Identifying eye movement related areas in the frontal lobe has a long history, with microstimulation in monkeys producing the most clear-cut results. For humans, however, there is still no consensus about the location and the extent of the frontal eye field (FEF). There is also no simple non-invasive method for unambiguously defining the FEF in individual subjects, a prerequisite for clinical applications. Here we explore the use of magnetoencephalography (MEG) for the non-invasive identification and characterization of FEF activity in an individual subject.
\end{abstract}

Methods: We mapped human brain activity before, during and after saccades by applying tomographic analysis to MEG data. Statistical parametric maps and circular statistics produced plausible FEF loci, but no unambiguous definition for individual subjects. Here we first computed the spectral decomposition and correlation with electrooculogram (EOG) of the tomographic brain activations. For each of these two measures statistical comparisons were made between different saccades.

Results: In this paper, we first review the frontal cortex activations identified in earlier animal and human studies and place the putative human FEFs in a well-defined anatomical framework. This framework is then used as reference for describing the results of new Fourier analysis of the tomographic solutions comparing active saccade tasks and their controls. The most consistent change in the dorsal frontal cortex was at the putative left FEF, for both saccades to the left and right. The asymmetric result is consistent with the 1-way callosal traffic theory. We also showed that the new correlation analysis had its most consistent change in the contralateral putative FEF. This result was obtained for EOG latencies before saccade onset with delays of a few hundreds of milliseconds (FEF activity leading the EOG) and only for visual cues signaling the execution of a saccade in a previously defined saccade direction.

Conclusions: The FEF definition derived from microstimulation describes only one of the areas in the dorsal lateral frontal lobe that act together to plan, prepare and execute a saccade. The definition and characterization of these areas in an individual subject can be obtained from non-invasive MEG measurements.

\section{Background}

Saccadic eye movements are quick, simultaneous movements of both eyes in the same direction. In the awake state saccades enable the eye to fixate sequentially on parts of the visual field. During the pre-saccadic period the eyes must be exactly coordinated to keep fixating on one place in the visual field, and then quickly the eyes

\footnotetext{
* Correspondence: a.ioannides@humanbraindynamics.com

'Laboratory for Human Brain Dynamics, AAl Scientific Cultural Services Ltd.,
} Nicosia, Cyprus are moved so that they land in synchrony on the same part of the visual field. In addition visual information from the old and new fixations must be integrated to provide the continuity of perception that characterizes primate vision. It is therefore no surprise that large areas of the cortex, mid-brain, cerebellum and brainstem are all part of the oculomotor system [1]. When saccades are cued by an auditory or visual cue they usually start a few hundreds of milliseconds after the cue onset and typically last less than $100 \mathrm{~ms}$. Here we studied the

\section{Biomed Central}


saccade-related eye fields in the frontal lobes of the brain. These areas lead the hierarchy of the oculomotor system and play a critical role in planning and initiating the eye movements. They are therefore sensitive indicators of the integrity of brain function in general and the frontal lobe in particular. Psychiatric disorders, including schizophrenia and depression are associated with eye movement dysfunction, especially during sleep [2]

Early studies have indicated that eye movements can be elicited by electrical stimulation of large parts of the frontal lobe in both monkeys $[3,4]$ and humans $[5,6]$. The development of microstimulation methods allowed "precise" definition of eye movement areas in the frontal cortex. One particular area, the Frontal Eye Field (FEF), is well defined anatomically, cytoarchitecturally and functionally in monkeys. The FEF is on the rostral bank of the arcuate sulcus and its stimulation with currents below $50 \mu \mathrm{A}$ produces saccades in the contralateral direction [7-9].

The introduction of positron emission tomography (PET) in the 1980s and functional Magnetic Resonance Imaging (fMRI) in the 1990s provided new tools for studying the human brain in general and saccadic eye movements in particular. Early studies identifying the "human FEF" produced consistent locations for PET $[10,11]$ and fMRI [12-14]. Surprisingly, this putative human FEF was identified more caudal and dorsal than would have been expected from cytoarchitectonic arguments and homology with the classic FEF established by the previous meticulous animal microstimulation experiments. New experiments with fMRI in monkeys $[15,16]$ and electrical stimulation in the lateral frontal cortex of epileptic patients $[17,18]$ resolved the paradox. For both monkeys and humans there are at least two areas associated with eye movements in the dorsal and lateral part of the frontal cortex [19]. One corresponds to the classic FEF, defined by its ability to elicit eye movements in the contralateral direction with low current injections. The other one is more caudal and superior, seen best with fMRI in both humans and monkeys.

The classic FEF, from the above studies, although undoubtedly an important structure, is only one of the areas in the dorsal part of the frontal lobe making up the network for the planning, control and initiation of eye movements. The studies reviewed above also suggested that there is a fairly strict homology between the structures identified in monkeys and humans. In this work we aimed to define the human homologue of the classic FEF and possibly other eye movement areas in the immediate vicinity of the dorsal and lateral aspects of the cortex. We will not consider the eye field areas close to the midline, namely the cingulate eye field [20] and supplementary eye field [21], or the areas in the more ventral part of the lateral frontal cortex. Here we will focus on the area that is roughly in front of the dorsal part of the motor cortex, at the level of the hand motor area. This is the area where, as we described above, recent studies in monkeys and humans have placed the two main frontal eye field areas.

From the theoretical point of view, the location and characterization of the human eye fields has its own intrinsic scientific importance. Added to this a better understanding of the role of each eye field may help us understand pathological conditions that are associated with changes in eye movement properties, and so possibly lead to clinical applications. It is well known that depressive illnesses, bipolar disorder, schizophrenia [22] and dementia [23] produce changes in eye movements. What is not clear is the frontal cortical component to these alterations, and how this may change with the severity of the disease and the likely therapeutic response to treatment. Neither a general definition of eye fields in terms of average locations across subjects nor a definition based on invasive stimulation is useful for treating individual patients. This is particularly important when considering epilepsy surgery for foci in the frontal cortical area. Detailed knowledge of the exact location of the eye movement fields in that patient will prevent damage to these structures. To this end, a noninvasive definition of the various eye fields for each individual subject is necessary. In this work we have taken a first step in this direction. Our starting point is the framework made up of human anatomical landmarks as defined by recent meta-analytic approaches linking monkey and human results $[17,19]$. We then place in the same framework the FEF loci defined by neuroimaging studies including our earlier MEG studies of saccadic eye movements $[1,24]$. Finally we show new and detailed analysis of a set of high sampling MEG recordings from one subject performing saccadic tasks. We present the results in the anatomical context established by the earlier studies. The new analysis identifies highly significant activations at loci corresponding to the FEFs defined by cortical stimulation and fMRI experiments. Our results reported here offer the first non-invasive clear-cut definition of FEFs in an individual subject and provide a rich characterization of the evolution of their activity over time during different eye movement tasks.

\section{Methods \\ Anatomical landmarks for the definition of FEF in earlier studies}

The recent studies reviewed in the Introduction have identified at least two "human FEFs". One corresponds to the classic FEF definition obtained by microstimulation in the monkey (and more recently in humans). The other is identified more prominently in fMRI studies from both humans and monkeys. We summarize these 
results and show them superimposed on the MRIs of one of our subjects using the key anatomical landmarks as references. The loci from the different studies are first transformed to a common Talairach space and then back transformed on to the MRI of the same subject. We are thus able to define the important sulci with the results of previous studies from our groups and others. The results of this new analysis can then be interpreted in the context of the earlier studies.

\section{Experiments and subjects}

The details for the two main MEG experiments (EXP1 and EXP2) studying saccades are described elsewhere $[1,24]$ and so are only briefly discussed here. In EXP1 the MEG signal was recorded for three types of saccades with a sampling rate of $625 \mathrm{~Hz}$ [1]. The first two saccade types were in the waking state, one following an auditory cue every 4 seconds and the other self initiated with the same tempo. We chose the tempo of one saccade every 4 seconds because it appeared easy and "natural". The third type was composed of saccades during rapid eye movement (REM) sleep. REM saccades were chosen from a whole-night MEG recording with properties similar to those studied in the awake state. The data from three subjects for the three types of saccades have been analyzed and the results reported in two publications $[1,25]$.

EXP2 was designed to disentangle aspects of saccades that could not be separated by the design of EXP1. EXP2 consisted of two separate sets of measurements. In the first set of measurements (EXP2A) four subjects were recorded with the same sampling rate of $625 \mathrm{~Hz}$ as in EXP1. An eye tracker and EOG were used to record the ocular muscle activity and the eye movements. In EXP2A we used a set of visual cues to specify whether a saccade was to be made or not (Move, M-cue; $\mathrm{GO} / \mathrm{NOGO}$ ), the direction of the saccade (Direction, $\mathrm{D}$-cue; Left/Right) or the initiation of the saccade (Action, A-cue). The $(\mathrm{M}+\mathrm{D}+\mathrm{A})$ information was provided at the same or different time, either in one step by a single cue, or in three steps separated by a few seconds from each other. There were five separate types of temporal sequences, one control condition (passive viewing of stimuli; Type0) and four saccade conditions (Type1-4). In the first two active sequences (Type1-2), the $\mathrm{M}, \mathrm{D}$ and $\mathrm{A}$ cue were presented with a few seconds between each other: in Type1 the sequence was $\mathrm{D} \rightarrow \mathrm{M}$ $\rightarrow \mathrm{A}$ and in Type 2 the sequence was $\mathrm{M} \rightarrow \mathrm{D} \rightarrow \mathrm{A}$. In the other two sequences (Type3-4) the information was collapsed into one cue. Type 3 sequence had only GO trials so the cue provided simultaneously the $(\mathrm{D}+\mathrm{A})$ information. Type 4 sequence had GO and NOGO trials so the cue provided simultaneously the full $(M+D+A)$ information. Of special interest and relevant to the results reported here are the cues releasing or inhibiting saccades (Cue3 for Type3-4, Cue6 for Type2), and the cue providing purely directional information (Cue4 in GO trials of Type2). A detailed description and diagrams of the stimuli and temporal sequences can be found in [24].

In the second part of EXP2 (EXP2B) we recorded the MEG signal from two subjects with a higher sampling rate of $2083 \mathrm{~Hz}$. To avoid lengthy experimental time and subject over-training, only a subset of sequences was used in EXP2B. In one subject, who also participated in EXP2A with simultaneous MEG, EOG and eye tracker recordings, we used sequences of Type0 (control) and Type3-4. No eye tracking was used on this occasion. For the second subject (did not participate in EXP2A), we recorded MEG signals together with EOG for sequences of Type0, 2, 3 and 4, 2 runs for each type. In the present paper we will report only results from the new analysis of the data from the second subject (hbd050) in EXP2B.

All our subjects were healthy, right handed males, with normal visual acuity, binocular vision and normal optic fundi. They had no history of neurological or psychiatric illness or drug abuse.

\section{Quantitative tomographic analysis of brain activity}

The previous results discussed in this paper together with the results of the new analysis on a single subject rely on meta-analysis of tomographic estimates of activity extracted for each timeslice of each single trial of the MEG data. Magnetic field tomography (MFT) [26] was used throughout to compute these estimates of brain activity as described in our earlier studies [1,24]. Briefly MFT produces a tomographic estimate of the vector field for the current density vector $\mathbf{J}(\mathbf{r}, \mathbf{t})$ in the brain from each timeslice of data. For each timeslice, $t$, the continuous estimates of $\mathbf{J}(\mathbf{r}, \mathbf{t})$ are discretized and stored for further analysis at $17 \times 17 \times 17$ grid points covering the whole brain. At the sampling rate of $2083 \mathrm{~Hz}$ (sampling step of $0.48 \mathrm{~ms}$ ), and given that for each cue presentation the MFT solutions were obtained for one second before and one second after cue onset, about 4000 or 8000 tomographic maps of $\mathbf{J}(\mathbf{r}, \mathbf{t})$ were obtained for each single trial depending on whether one (Type0, 3-4) or two (Type2) cues were selected for the analysis. Clearly the volume of MFT solutions requires post-MFT analysis at different levels, such as averaging, statistical parametric mapping (SPM), circular statistics $[27,28]$ as previously described $[1,24]$. The SPM comparisons of different saccadic tasks produced well-circumscribed foci of activity. These foci guided further inspection of the single trial MFT solutions for the definition of regions of interest (ROI) and the dominant (main) direction of the current density vector. These ROIs were 
used to extract time series for regional activations in each single trial and hence provide time-dependent measures of signal content within areas (e.g. signal to noise ratio) or measures of linked activity between areas (e.g. mutual information) $[1,24]$. Detailed analysis was performed after alignment of the MFT solutions relative to either cue or saccade onset. Here we also introduced two new measures of brain activity: spectral analysis and correlations between brain activation and auxiliary EOG channel. Each of these two measures was applied independently for each grid point producing alternative tomographic representations of brain activity. We describe the two new measures next.

\section{Spectral analysis of single trial MFT solutions}

The current density vector $\mathbf{J}(\mathbf{r}, \mathrm{t})$ from MFT solution at each grid point produces three timeseries, one for each Cartesian component. The Fourier transform coefficients for each component are obtained from segments of duration $D$ (from $t_{1}$ to $t_{2}, D=t_{2}-t_{1}$ ) in a frequency range from $f_{1}$ to $f_{2}$ at a step $\Delta f$. At each frequency a pseudo-vector can be defined from the Fourier amplitude for each Cartesian component of $\mathbf{J}(\mathbf{r}, \mathrm{t})$. The moduli of these pseudo-vectors are then used to make grid point by point comparisons between conditions, using SPM in exactly the same way as for the time domain, but now describing the significant changes of activity within frequency ranges. In the Results section we will report the Fourier spectra extracted from two $400 \mathrm{~ms}$ long single trial segments aligned to saccade onset (defined by the EOG channel). The first segment was extracted just before saccades (from -410 to $-10 \mathrm{~ms}$ ). The second segment was centered at the saccade onset (from -200 to $200 \mathrm{~ms}$ ) and thus captured the entire saccade period, which usually lasts well below $100 \mathrm{~ms}$. For each of these two segments, the Fourier spectra were computed from $3 \mathrm{~Hz}$ to $600 \mathrm{~Hz}$ at a step of $1 \mathrm{~Hz}$. Then, we applied SPM analysis to compare two distributions. Each distribution consisted of the frequency amplitudes from a sliding $4-\mathrm{Hz}$ window (5 values from each single trial). The centre of the window covered the range from 5 to $593 \mathrm{~Hz}$ with a step of $2 \mathrm{~Hz}$. Statistical comparisons were made between each of the two recording runs for Type3, Type4 and the control condition (Type0), separately for saccades to the left and right. Finally, grid points showing common activations in the four SPM maps with significance level p $<0.005$ (after Bonferoni correction) were delineated and placed in the context of anatomical landmarks defined earlier. These common activations were identified within an $80 \mathrm{~Hz}$ window. The centre of this window was from $45 \mathrm{~Hz}$ to $545 \mathrm{~Hz}$ in 20 $\mathrm{Hz}$ steps.

\section{Correlations between brain activations and auxiliary EOG channel}

For each single trial aligned to the cue onset, we computed the cross correlation between brain activations and the EOG for segments of duration $D=200 \mathrm{~ms}$. The correlation was done for each current density component at each grid point with a relative delay, $\tau$, between the brain activity and EOG. This resulted in cross correlation vectors $\mathbf{C}(\tau)$. To remove the strong dependence of $\mathbf{C}$ on periods with large EOG or J values, we normalized $\mathbf{C}$ by dividing each of its components by the Euclidean norms of $\mathbf{J}(\mathbf{r}, \mathrm{t})$ and EOG segments over the time range of D. The normalized correlation vectors, $\hat{\mathbf{C}}$, were computed for $\tau$ from $-600 \mathrm{~ms}$ (i.e. brain activity leading the EOG) to $200 \mathrm{~ms}$ (i.e. brain activity lagging the EOG) with a step of $20 \mathrm{~ms}$. Then, we applied SPM analysis to compare two distributions made of moduli of normalized correlation coefficients $(\hat{\mathrm{C}}=|\hat{\mathbf{C}}|)$. The pair of distributions was from different cues of the same trial (e.g. Cue6 versus Cue4 in Type2) or same cue comparing GO with NOGO trials (e.g. Cue6 in Type2; Cue3 in Type4). The comparisons were computed for delays, $\tau$, from -580 to $+160 \mathrm{~ms}$ in steps of $20 \mathrm{~ms}$, allowing a delay jitter of $20 \mathrm{~ms}$. We will report results for two EOG reference segments: one is centered at 0 , corresponding to the cue image onset and hence well before any strong EOG activity associated with saccade onset. The other is centered at $300 \mathrm{~ms}$ post cue image onset, corresponding to the mean time of the saccade onset, and thus likely to contain at least in some of the trials strong EOG activity related to the saccade onset.

\section{Results}

\section{Characterization of FEF activity from our earlier studies}

From the EXP1 data, we identified consistent foci of activity on the cortex, cerebellum and brainstem, for three subjects. Specifically, some of the cortical activity was consistent with the FEF location as far as one could judge on the basis of the landmarks of the local anatomy of each subject. The activation time courses of each area were extracted from the tomographic solutions and analyzed in single trials. The analysis showed that the entire process of saccade planning, initiation and execution seemed to merge into the imposed $(0.25 \mathrm{~Hz})$ rhythm that dominated the activity in each area. A more detailed analysis of the data showed that slow rhythms and fast activity contributed to brain activations and the way that these activations were linked to each other and the eye movements (as these were quantified by the fast EOG activity) [1].

From the EXP2 data, for two subjects, we identified highly significant changes of activity in all the key areas, 
including putative FEF, cerebellum and brainstem. Thanks to the higher sampling rate than EXP1 and the use of sequences with cues providing separate pieces of information about the saccadic task, we were able to show that the brain used each piece of information as soon as it was available using both fast transient activity, lasting only a few milliseconds, and slow activity, lasting hundreds of milliseconds [24].

\section{Anatomical and functional definition of human FEF}

The human FEF as defined in a series of recent studies using epicortical stimulation [17], fMRI [19,29,30], fMRI-MEG [31] and MEG [1,24] are shown on subject hbd050's MRIs (Figure 1). In this figure, we used the anatomical landmarks as described in [19], the MNI and/or Talairach coordinates for FEF definition provided in the above five studies by others $[17,19,29-31]$ and our two studies $[1,24]$. The loci defined in the Talairach space were then back-transformed to the MRI coordinates of subject hbd050 for the final display. The display is shown at two axial slices, with Talairach coordinate of (a) $\mathrm{Z}=45 \mathrm{~mm}$ and (b) $\mathrm{Z}=37 \mathrm{~mm}$, respectively. The major sulci are represented in the same figure by heavy white outlines: the superior frontal sulcus (SFS) runs from the rostral end of each hemisphere in the anterior-posterior direction, while the pre-central (PCS) and central sulci (CS) run along a lateral to medial direction with the
PCS the more anterior of the two. We use color to distinguish the FEF definitions from the seven studies. For each of the studies by others, we represent the FEF definitions by the mean location averaged across subjects for each hemisphere. For our studies, we use the FEF definitions for each of the five subjects (three subjects from EXP1 and two from EXP2, left and right hemisphere, so total of 10 FEF locations). Figure 1 shows that seven out of 10 loci either coincide or are close to the border of the definition provided by Blanke et al. [17], while one more is just behind the precentral sulcus (yellow circle in the left hemisphere in Figure 1b) and it is therefore more consistent with the FEF definition usually given by fMRI studies. The other two FEF definitions were up to $1 \mathrm{~cm}$ more inferior to the axial slice in Figure $1 \mathrm{~b}$. In summary our post-MFT analysis produced FEF definitions that were broadly consistent with those derived from epicortical stimulations.

\section{New FEF definitions}

In our two earlier studies on eye movement, we identified activity in many other parts of the frontal cortex, not just the putative FEFs discussed above, at different times before, during and after saccades. The high variability across trials and subjects prompted us to look for quantitative descriptions of activity in the frequency domain using the procedure described in the Methods

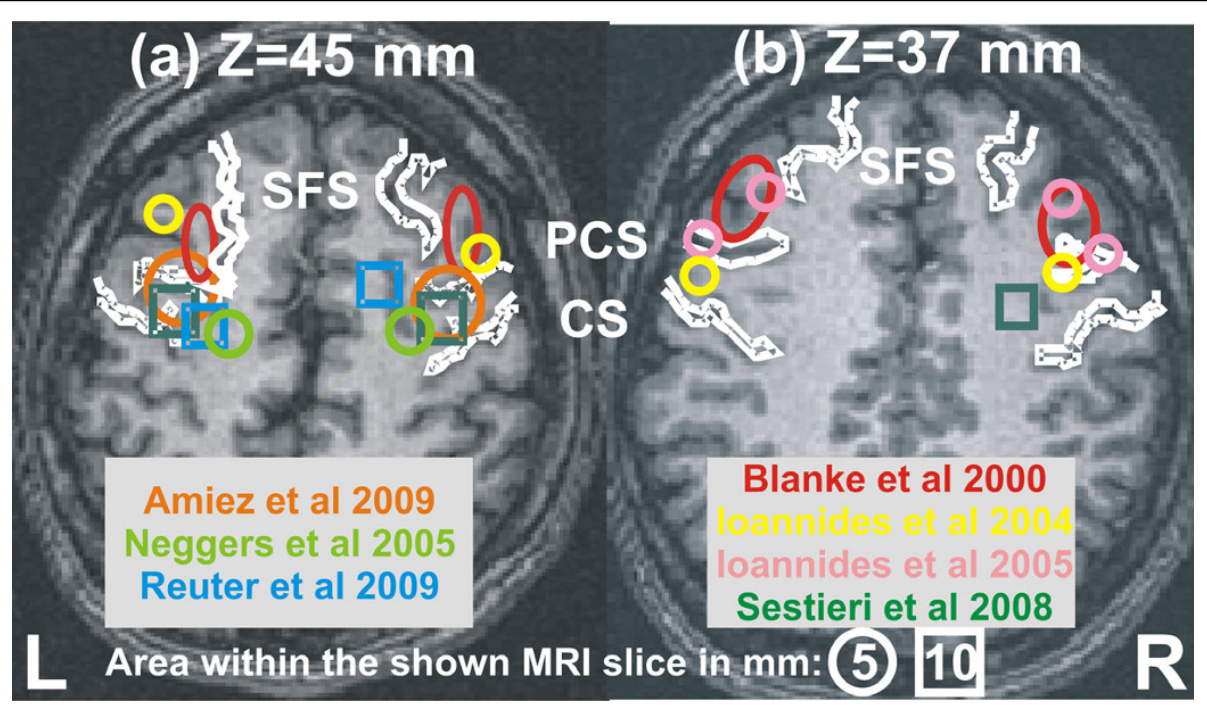

Figure 1 Definition of human FEF from seven studies. The FEF loci were transformed into the Talairach space and superimposed onto one of our subjects' MRIs. The display is shown at two axial slices, one with Talairach coordinate of (a) $\mathrm{Z}=45 \mathrm{~mm}$ and one with (b) Z=37 mm. Color denotes the FEF definition from different studies (Red: Blanke et al [17]); orange: Amiez et al [19]; light green: Neggers et al [29]; green: Sestieri et al [31]; blue: Reuter et al [30]; yellow: loannides et al 2004 [1] and pink: loannides et al 2005 [24]). Shape denotes the distance between the loci and the MRI slices (circles and squares for loci within $5 \mathrm{~mm}$ and $10 \mathrm{~mm}$ of the slice, respectively). For reference, major landmarks are also shown as white outlines: central sulcus (CS), precentral sulcus (PCS) and superior frontal sulcus (SFS). The FEF definitions from each of the studies by others are represented by one set of symbols (one for left and one for right hemisphere, each marking the mean coordinates of a group of subjects used in that study), while from our studies the FEF definitions for individual subjects are shown (yellow circles in (a), yellow and pink circles in (b)). 
section. Statistical comparisons of the Fourier spectra between saccades and passive viewing produced clear left FEF activation for both the pre- and peri-saccadic periods. Saccade data were from Type3 and Type4, 2 runs for each Type, while passive viewing is from Type0, so there are four comparisons in total (i.e. Type3 vs. Type0; Type4 vs. Type0). Figure 2 shows the common significant change of activity from the four comparisons with a center window at $125 \mathrm{~Hz}$ (i.e. window range 85 to $165 \mathrm{~Hz}$ ), for $10^{\circ}$ saccades from the center to the left (left column) and to the right (right column). The figure shows the FEF loci spreads from the classic FEF location (as defined by Blanke et al. [17]; dotted magenta ellipsoids in Figure 2) to a more posterior area for saccades to the right, especially during the saccade period (Figure 2b). These results were identified in the same area (left FEF) over a wide range of frequencies, from $85 \mathrm{~Hz}$ to $500 \mathrm{~Hz}$ for saccades to the left and from 85 to $545 \mathrm{~Hz}$ (the highest frequency window studied) for saccades to the right.

The definition for FEF for the single subject is best obtained from the correlation measure: contralateral FEF activity was observed clearly with delay relative to the EOG of a few hundred milliseconds (brain activity leading the EOG). As an example, we computed the correlation between brain activity and EOG for Cue4 and Cue6 from Type2 GO trials. In Type 2 runs, the saccade information is given in the order of M, D and A (Move, Direction, Action cues; see Methods). Both Cue4 and Cue6 mark the onset of the same visual image but carry different saccade information to the subject. Cue4 indicates the saccade direction, while Cue6 is the action cue

(a) pre-saccadic period: -410 to $-10 \mathrm{~ms}$ relative to saccade onset

(b) peri-saccadic period: -200 to $200 \mathrm{~ms}$ relative to saccade onset

(c) periods from

(a) and (b)

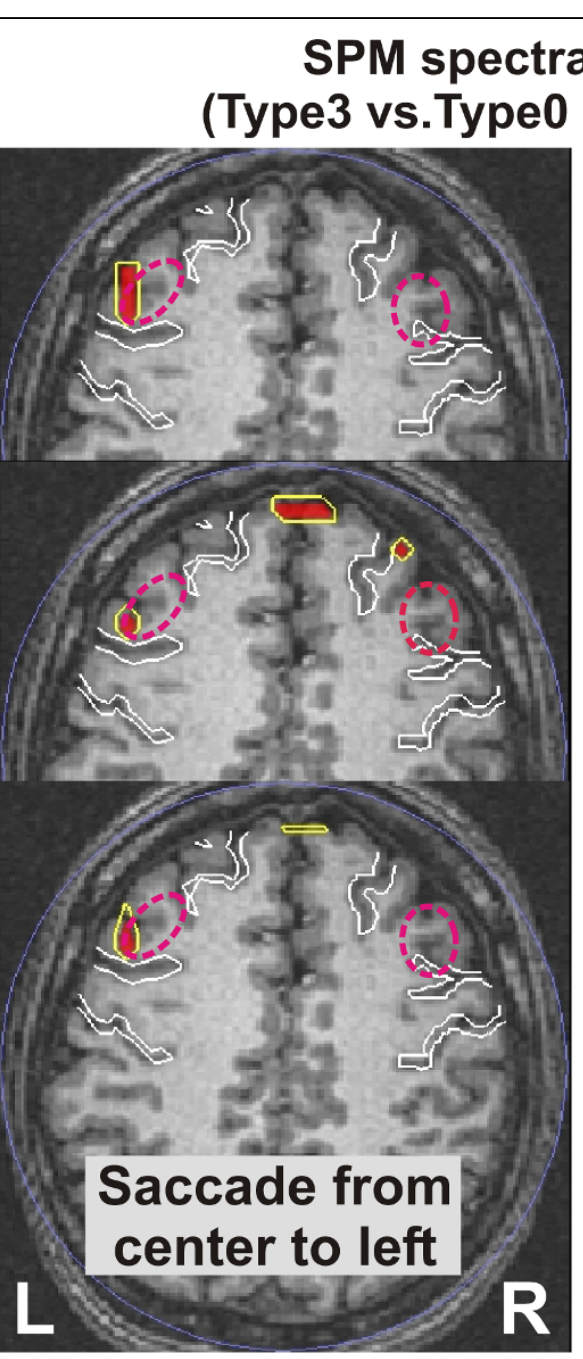

SPM spectra comparisons (Type3 vs.Type0 + Type4 vs.Type0)

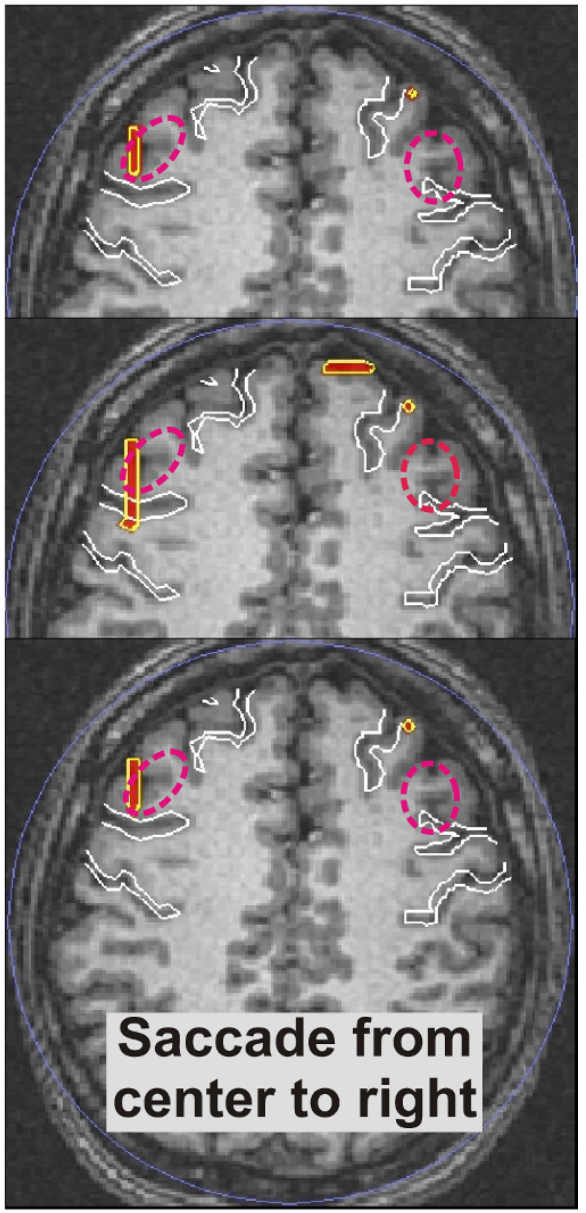

Figure 2 Statistical comparison of spectra between saccades and passive viewing. Common significant change of activity from saccades (Type3, Type4) versus passive viewing (Type0) for pre-saccadic (a), peri-saccadic (b) and both (c) periods, respectively for saccades to the left (left column) and to the right (right column). Red blob and yellow contours denote areas with $p<0.005$. Dotted magenta ellipsoid marks the classic FEF as defined by Blanke's microstimuation study in humans [17], while white outlines mark major landmarks as in Figure 1. 
that subject should saccade as soon as this cue is present. We then applied the SPM analysis to the normalized correlation coefficients for Cue4 and Cue6. Figure 3 shows the SPM results (yellow contours) from the EOG reference segment centered at the cue image onset (see Methods), for saccades to the left (a) to the right (b). The figure shows clear-cut contralateral FEF loci, close to the classic FEF location (dotted magenta ellipsoids in Figure 3). Notably, in Figure 3a, as the delay between brain and EOG activity $(\tau)$ is reduced, right FEF spreads from a rostral $(\tau=-280 \mathrm{~ms})$ to more posterior area $(\tau=-160 \mathrm{~ms})$. This posterior area is usually defined as FEF from fMRI studies (see Figure 1a). Furthermore, similar results are observed: (1) When the EOG reference segment is centered at $300 \mathrm{~ms}$ post cue image onset, which corresponds roughly to the mean time of saccade onset and thus likely to contain some strong EOG activity related to the saccade onset (see Methods); (2) For comparison of Cue6 GO versus NOGO trials in Type2. Importantly, the above result is not seen for the comparison between the Cue $3 \mathrm{GO}$ and NOGO trials in Type4. Our results thus suggest that contralateral FEF activity is present only if the directional information is already given to the subject and the subject maintains the ocular segments in readiness for this goal while waiting for the action cue to make a saccade.

\section{Discussion}

The overall picture that emerges from our MEG studies is a very complex one, but consistent with both the microstimulation and fMRI studies. In our previous studies, we found no simple way to define the FEFs for individual subjects: the definition cannot be guided by the latency or the laterality of the activations obtained by averaging across trials, nor from the SPM comparisons between conditions in any specific latency range in the few hundreds of milliseconds before, during or after saccade onset. Although it was possible to obtain what in retrospect appeared to be good FEF definitions (purely judging on anatomical criteria), the procedure was so cumbersome and complicated (see subsection Complex identification of regions of interest in the Methods section in [24]) that it cannot be routinely used in a clinical setting. There was no obvious simple sequence in the time domain that could capture adequately the variance in the single trial activations. The most ordered

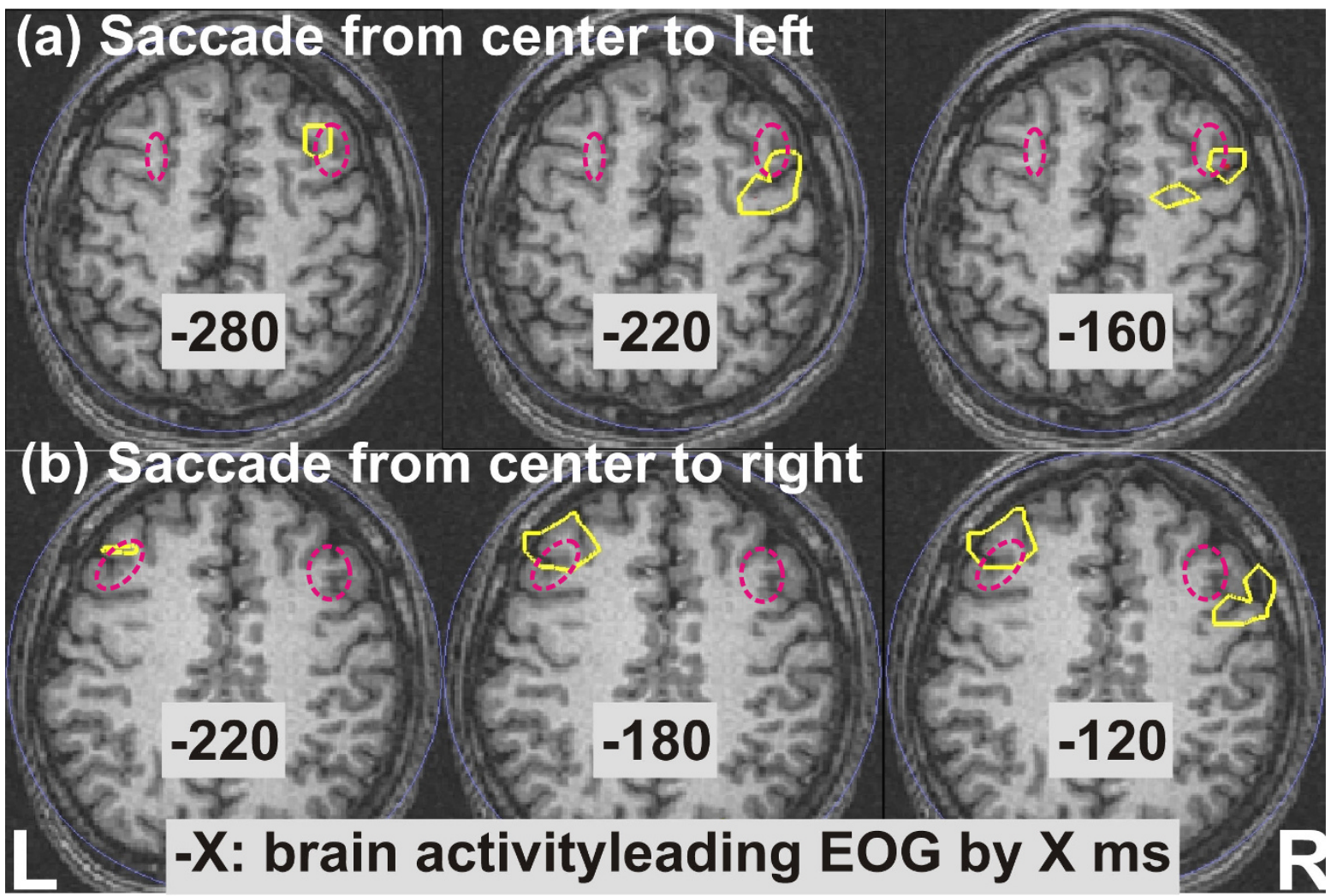

Figure 3 Statistical comparison of correlations between brain activity and EOG. The computation is made from Type 2 GO trials, Cue 6 (action cue) versus Cue4 (direction cue), for saccades to the left (a) and to the right (b). Results for different time delays between the brain activity and EOG are shown in different columns. Yellow contours denote the statistical significance threshold of $p<0.001$. Dotted magenta ellipsoid marks the classic FEF location [17]. 
set of activations in our earlier studies, and also consistent across subjects, were obtained when the activity in different brain areas was linked to the EOG using mutual information (MI) analysis [1]. The MI was computed relative to EOG onset and as a function of delay between brain and EOG activations. FEF activity in the last $100 \mathrm{~ms}$ leads to peaks in MI corresponding to EOG activity after EOG onset. The brain activity just before saccade onset, even at the level of the brain stem gaze centers, was linked to EOG burst after saccade onset [1]. The variability across single trials was even more evident in the analysis of the second experiment. In this experiment too, the MI analysis between brain areas provided the best organizing principle for the sequence of activations [24]. In addition circular statistics analysis of fast transient responses showed that the even at the finest timescales (millisecond), we could access a rich organization that could not be adequately described by simple stacking of single trials across external markers like cue or saccade onset.

The more refined analysis reported here for a single subject begins to unravel some of the complexities associated with the role of the FEF in saccade generation. The frequency analysis shows that the changes in presaccadic and peri-saccadic periods compared to passive viewing of stimuli are more prominent in the left FEF for both saccades to the left and right. The one-way callosal traffic theory [32] offers one possible explanation for this asymmetry. According to this theory the decision to move the eyes begins in the major hemisphere, i.e. left hemisphere for a right-handed subject. The excess activity in the left FEF compared to the control condition could therefore be interpreted as the correlate of the decision to execute the eye movement by the left hemisphere.

The contralateral FEF is best seen in the correlation between brain activations and the EOG. The laterality of the FEF activation is not just on the contralateral side, as shown by the Fourier spectra analysis, so the purely contralateral activity is most clearly seen when the directional information is already provided and the subject simply waits for the action cue. The result is therefore clearer for comparisons between periods before a cue signaling the execution of a saccade in a given direction with periods before a cue providing information about which direction the saccade will be made (Figure 3) or when the same cue is from a NOGO trial.

Based on our results we can interpret the most widely used definition of FEF (through microstimulation experiments) in a new way. This definition fit the data because it bypasses many of the complexities that voluntary and cued saccades necessarily entail. Eye movement is the outcome of finely balanced competing actions by agonist and antagonist muscles [33]. The classic definition of FEF should then be interpreted as the identification of the cortical brain area where minimal injection of current can tip the balance in favor of a saccade.

On the other hand, the functional activations from fMRI have identified a more general region, which is likely to be involved in a more complex task than just "tipping the balance". We know from our previous study [24] that the activity in the oculomotor system depends on linked activity at different scales, from a few milliseconds to a good fraction of a second. It is therefore impossible to study saccades in detail because of the limited time resolution of the technique. In addition to the lack of millisecond time resolution, the sluggishness of the fMRI response means that it can never isolate the activity related to saccades in just one direction. This is because after each saccade, the eye must either be held with gaze away from equilibrium or a movement must be made to return the eye to the centre. Each of these actions is likely to modify the activity in the same areas involved in the saccade generation. Our new analysis allows us to characterize the saccade-related activity within finite latency ranges using different time scales (frequencies) and isolate the part of the activity that relates to the EOG. Our new results suggest a rostro-caudal progression of the activity as the time of the saccade initiation approaches, beginning with activity in the classic FEF area as defined by microstimulation progressing later to the more posterior areas that are more prominently seen with fMRI.

We note also that saccade onset can be delayed by transcranial magnetic stimulation (TMS) [34], but it has been proved more difficult to initiate saccades using TMS. This may be a consequence of the complex spatial organization that the rather crude TMS stimulation does not fulfill, unlike microstimulation that will selectively target a discrete area. By exciting a large area TMS is likely to stimulate cells initiating and inhibiting saccades.

As in our other two studies [1,24] the lag between the FEF activity and activity in the ocular muscles, as measured by the EOG, is measured in hundreds of milliseconds and it is present already before the cue image onset and it continues through to the saccade onset (data not shown). The EOG activity before saccade onset is usually considered as random fluctuations. Our results show that this pre-saccadic EOG activity is clearly correlated with task-related FEF activity. We speculate that this pre-saccadic EOG activity reflects the preparation of ocular muscles for accurate execution of the impending saccades. The linked activity between the EOG and the brain can be captured by linear measures like correlation or non-linear ones like MI, and thus provides a sensitive tool for exploring the role of specific areas in different saccadic tasks. This speculation is supported by our other study in this volume dealing with 
precise measurements of each eye's position when subjects executed saccade sequences of Type 3 and Type 4 [35]. The mutual information in that study was computed between timeseries for the location and velocity of each eye and thus the yoking of the two eyes was defined. Particularly relevant to our results reported here was the finding that before the initiation of saccades, the MI derived from the position information of the yoking of the two eyes was significantly higher in the Type4 (GO/NOGO trials) sessions relative to the Type3 (GO trials only) sessions.

\section{Conclusions}

It is possible to define the human $\operatorname{FEF}(\mathrm{s})$ of individual subjects after careful analysis of the tomographic estimates of activity extracted from completely non-contact, non-invasive MEG recordings. The use of time domain information leads to the identification of either the homologue of the classic FEF identified in animals or the "other" FEF located a little more caudally and dorsally. Adding frequency analysis and especially the grid point by point correlation with the EOG can separate these two FEF subdivisions (and probably show further subdivisions). In this study we emphasized the localization and characterization of the FEF for a single subject. The generalization of the specific results we reported here cannot be assumed $a$ priori for other subjects. It is likely that some of the details may change, so to reach conclusions for the population the experiments must be repeated with many subjects, taking into account the age, gender, and handedness of individual subjects.

A long term goal of our work is to provide characterization of the state of an individual and biomarkers from non-invasive measurements. Eye movements are known to be affected in many neurological and psychiatric disorders. We anticipate that clinically useful biomarkers can be defined for application to individual subjects by combining a detailed understanding of the oculomotor system anatomy and function with detailed observations of its electric and magnetic activity and even just optical recordings. The results reported here and in the companion study [35] represent the first tentative steps in this direction. For future use in clinical applications it is important to identify how reproducible these definitions are for the same individual across weeks, months and years, so that the FEF localization and functional characterization can serve as a biomarker of health and disease.

\section{Acknowledgements}

The MEG data of the present study was collected from the Laboratory for Human Brain Dynamics in RIKEN Brain Science Institute, Japan. The data was analyzed in the Laboratory for Human Brain Dynamics, AAI Scientific Cultural Services Ltd, Cyprus. A grant from Cyprus Research Promotion Foundation,
"Hosting of Researchers Based Abroad" (no. 0308/09), provided the financial support for PBCF to work in Cyprus.

This article has been published as part of Nonlinear Biomedical Physics Volume 4 Supplement 1, 2010: Consciousness and its Measures: Joint Workshop for COST Actions Neuromath and Consciousness. The full contents of the supplement are available online at http://www.nonlinearbiomedphys.com/supplements/4/S1

\section{Author details}

'Laboratory for Human Brain Dynamics, AAI Scientific Cultural Services Ltd., Nicosia, Cyprus. ${ }^{2}$ Kings College Institute of Psychiatry, London, UK.

\section{Authors' contributions}

AAl, LCL and PBCF designed the experiment and carried out the MEG measurements. AAI and LCL conducted the data analysis. AAI, PBCF and EP worked on the anatomical definitions of FEFs and the representation of functional definitions of FEFs from previous studies on common diagrams. $\mathrm{AAI}, \mathrm{LCL}$ and $\mathrm{EP}$ designed and produced the figures. AAI and $\mathrm{LCL}$ wrote the manuscript. All authors read and approved the final manuscript.

\section{Competing interests}

The authors declare that they have no competing interests.

Published: 3 June 2010

\section{References}

1. Ioannides AA, Corsi-Cabrera M, Fenwick PBC, Portilla YD, Laskaris NA, Khurshudyan A, Theofilou D, Shibata T, Uchida S, Nakabayashi T, et al: MEG tomography of human cortex and brainstem activity in waking and REM sleep saccades. Cerebral Cortex 2004, 14:56-72.

2. Benson KL, Zarcone VP Jr: Rapid eye movement sleep eye movements in schizophrenia and depression. Arch Gen Psychiatry 1993, 50:474-482.

3. CROSBY EC, YOSS RE, HENDERSON JW: The mammalian midbrain and isthmus regions. Part II. The fiber connections. D. The pattern for eye movements on the frontal eye field and the discharge of specific portions of this field to and through midbrain levels. J Comp Neurol 1952, 97:357-383.

4. Ferrier D: Experiments on the basis of monkeys. Proc R Soc Lond B Bio Sci 1875, 23:409-430.

5. Foerster O: The cerebral cortex in man. Lancet 1931, 2:309-312.

6. Penfield W, Rasmussen T: The Cerebral Cortex of Man. A Clinical Study of Localization of Function. McMillan 1950.

7. Bruce CJ, Goldberg ME: Primate frontal eye fields. I. Single neurons discharging before saccades. J Neurophysiol 1985, 53:603-635.

8. Bruce CJ, Goldberg ME, Bushnell MC, Stanton GB: Primate frontal eye fields. II. Physiological and anatomical correlates of electrically evoked eye movements. J Neurophysiol 1985, 54:714-734.

9. Stanton GB, Deng SY, Goldberg ME, McMullen NT: Cytoarchitectural characteristic of the frontal eye fields in macaque monkeys. J Comp Neurol 1989, 282:415-427.

10. Paus T: Location and function of the human frontal eye-field: a selective review. Neuropsychologia 1996, 34:475-483.

11. Petit L, Dubois S, Tzourio N, Dejardin S, Crivello F, Michel C, Etard O, Denise P, Roucoux A, Mazoyer B: PET study of the human foveal fixation system. Hum Brain Mapp 1999, 8:28-43.

12. Corbetta M, Akbudak E, Conturo TE, Snyder AZ, Ollinger JM, Drury HA, Linenweber MR, Petersen SE, Raichle ME, Van Essen DC, et al: A common network of functional areas for attention and eye movements. Neuron 1998, 21:761-773.

13. Luna B, Thulborn KR, Strojwas MH, McCurtain BJ, Berman RA, Genovese CR, Sweeney JA: Dorsal cortical regions subserving visually guided saccades in humans: an fMRI study. Cereb Cortex 1998, 8:40-47.

14. Petit L, Clark VP, Ingeholm J, Haxby JV: Dissociation of saccade-related and pursuit-related activation in human frontal eye fields as revealed by fMRI. J Neurophysiol 1997, 77:3386-3390

15. Baker JT, Patel GH, Corbetta M, Snyder LH: Distribution of activity across the monkey cerebral cortical surface, thalamus and midbrain during rapid, visually guided saccades. Cereb Cortex 2006, 16:447-459.

16. Koyama M, Hasegawa I, Osada T, Adachi Y, Nakahara K, Miyashita Y: Functional magnetic resonance imaging of macaque monkeys 
performing visually guided saccade tasks: comparison of cortical eye fields with humans. Neuron 2004, 41:795-807.

17. Blanke O, Spinelli L, Thut G, Michel CM, Perrig S, Landis T, Seeck M: Location of the human frontal eye field as defined by electrical cortical stimulation: anatomical, functional and electrophysiological characteristics. Neuroreport 2000, 11:1907-1913.

18. Yamamoto J, Ikeda A, Satow T, Matsuhashi M, Baba K, Yamane F, Miyamoto S, Mihara T, Hori T, Taki W: Human eye fields in the frontal lobe as studied by epicortical recording of movement-related cortical potentials. Brain 2004, 127:873-887.

19. Amiez C, Petrides M: Anatomical organization of the eye fields in the human and non-human primate frontal cortex. Prog Neurobiol 2009, 89:220-230.

20. Dum RP, Strick PL: The origin of corticospinal projections from the premotor areas in the frontal lobe. J Neurosci 1991, 11:667-689.

21. Brinkman C, Porter R: Supplementary motor area in the monkey: activity of neurons during performance of a learned motor task. J Neurophysiol 1979, 42:681-709.

22. Harris MS, Reilly JL, Thase ME, Keshavan MS, Sweeney JA: Response suppression deficits in treatment-naive first-episode patients with schizophrenia, psychotic bipolar disorder and psychotic major depression. Psychiatry Res 2009, 170:150-156.

23. Crutcher MD, Calhoun-Haney R, Manzanares CM, Lah JJ, Levey Al, Zola SM: Eye tracking during a visual paired comparison task as a predictor of early dementia. Am J Alzheimers Dis Other Demen 2009, 24:258-266.

24. Ioannides AA, Fenwick PB, Liu L: Widely distributed magnetoencephalography spikes related to the planning and execution of human saccades. J Neurosci 2005, 25:7950-7967.

25. Ioannides AA, Kostopoulos GK, Liu L, Fenwick PB: MEG identifies dorsal medial brain activations during sleep. Neuroimage 2009, 44:455-468.

26. Ioannides AA, Bolton JPR, Clarke CJS: Continuous probabilistic solutions to the biomagnetic inverse problem. Inverse Problems 1990, 6:523-542.

27. Fisher NI: Statistical analysis of circular data. Cambridge University Press 1993.

28. Mardia KV, Jupp PE: Directional statistics. Wiley 2000.

29. Neggers SF, Raemaekers MA, Lampmann EE, Postma A, Ramsey NF: Cortical and subcortical contributions to saccade latency in the human brain. Eur J Neurosci 2005, 21:2853-2863.

30. Reuter B, Kaufmann C, Bender J, Pinkpank T, Kathmann N: Distinct Neural Correlates for Volitional Generation and Inhibition of Saccades. J Cogn Neurosci 2009

31. Sestieri C, Pizzella V, Cianflone F, Romani GL, Corbetta M: Sequential activation of human oculomotor centres during planing of visuallyguided eye movement: a combined fMRI-MEG study. Frontiers in Human Neuroscience 2008, 1:1-8.

32. Derakhshan I: How do the eyes move together? New understandings help explain eye deviations in patients with stroke. CMAJ 2005, 172:171-173.

33. Leigh RJ, Zee DS: The Neurology of Eye Movements. Oxford University Press 1999, 215-217.

34. Priori A, Bertolasi L, Rothwell JC, Day BL, Marsden CD: Some saccadic eye movements can be delayed by transcranial magnetic stimulation of the cerebral cortex in man. Brain 1993, 116(Pt 2):355-367.

35. Maruyama M, Fenwick PBC, loannides AA: Interocular yoking in human saccades examined by mutual information analysis. Nonlinear Biomedical Physics 2010, 4(Suppl 1):S10.

doi:10.1186/1753-4631-4-S1-S11

Cite this article as: loannides et al:: A step towards non-invasive characterization of the human frontal eye fields of individual subjects. Nonlinear Biomedical Physics 2010 4(Suppl 1):S11.

\section{Submit your next manuscript to BioMed Central and take full advantage of:}

- Convenient online submission

- Thorough peer review

- No space constraints or color figure charges

- Immediate publication on acceptance

- Inclusion in PubMed, CAS, Scopus and Google Scholar

- Research which is freely available for redistribution

Submit your manuscript at www.biomedcentral.com/submit
Biomed Central 